\title{
Catalytic Growth and Characterization of Gallium Nitride Nanowires
}

\author{
Chia-Chun Chen, ${ }^{*},{ }^{\dagger}$ Chun-Chia Yeh, ${ }^{\ddagger}$ Chun-Ho Chen, ${ }^{\dagger}$ Min-Yuan Yu, ${ }^{\dagger}$ Hsiang-Lin Liu, ${ }^{\S}$

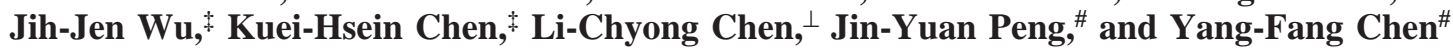

\begin{abstract}
Departments of Chemistry and Physics, National Taiwan Normal University, 88 s. 4 Tingchow Rd., Taipei 116, Taiwan, Institute of Atomic and Molecular Sciences, Academic Sinica, Taipei, Taiwan, Center for Condensed Matter Science and Department of Physics, National Taiwan University, Taipei, Taiwan
\end{abstract}

Received November 22, 2000

\begin{abstract}
The preparation of high-purity and -quality gallium nitride nanowires is accomplished by a catalytic growth using gallium and ammonium. A series of catalysts and different reaction parameters were applied to systematically optimize and control the vapor-liquid-solid (VLS) growth of the nanowires. The resulting nanowires show predominantly wurtzite phase; they were up to several micrometers in length, typically with diameters of 10-50 nm. A minimum nanowire diameter of $6 \mathrm{~nm}$ has been achieved. Temperature dependence of photoluminescence spectra of the nanowires revealed that the emission mainly comes from wurtzite GaN with little contribution from the cubic phase. Moreover, the thermal quenching of photoluminescence was much reduced in the GaN nanowires. The Raman spectra showed five first-order phonon modes. The frequencies of these peaks were close to those of the bulk GaN, but the modes were significantly broadened, which is indicative of the phonon confinement effects associated with the nanoscale dimensions of the system. Additional Raman modes, not observed in the bulk GaN, were found in the nanowires. The field emission study showing notable emission current with low turn-on field suggests potential of the GaN nanowires in field emission applications. This work opens a wide route toward detailed studies of the fundamental properties and potential applications of semiconductor nanowires.
\end{abstract}

\section{Introduction}

One-dimensional (1-D) semiconductor nanostructures (nanorods and nanowires) have recently brought significant research activities in material chemistry. ${ }^{1}$ Nanorods and nanowires are intriguing 1-D structural and electronic-confined systems ideally suited for fundamental studies of their physical properties and for the fabrication of optoelectronic nanodevices. ${ }^{2}$ Notably, one of the 1-D III-V semiconductor nanostructures, GaN nanowires, has attracted much attention because of their great potential for the new visible and UV optoelectronic applications. ${ }^{3}$ Of late, the synthesis of GaN nanowires has been achieved by various techniques such as laser-ablation, ${ }^{4}$ carbon-nanotube-confined

\footnotetext{
* To whom correspondence should be addressed. E-mail: t42005 @ cc.ntnu.edu.tw.

Department of Chemistry, National Taiwan Normal University.

Institute of Atomic and Molecular Sciences, Academic Sinica.

$\S$ Department of Physics, National Taiwan Normal University.

$\perp$ Center for Condensed Matter Science, National Taiwan University.

\# Department of Physics, National Taiwan University.

(1) (a) Alivisatos, A. P. Science 1996, 271, 933-937. (b) Lieber, C. M. Solid State Comm. 1998, 107, 607-616. (c) Collier, C. P.; Vossmeyer, T. Heath, J. R. Annu. Rev. Phys. Chem. 1998, 49, 371-404. (d) Dekker, C. Phys. Today 1999, 52, 22-28. (e) Fan, S.; Chapline, M. G.; Franklin, N. R.; Tombler, T. W.; Cassell, A. M.; Dai, H. Science 1999, 283, 512-514.

(2) (a) Peng, X.; Manna, L.; Yang, W.; Wickham, J.; Scher, E.; Kadavanich, A.; Alivisatos, A. P. Nature 2000, 404, 59-61. (b) Morales, A. M.; Lieber, C. M. Science 1998, 279, 208-211. (c) Colvin, V. L.; Schlamp, M. C.; Alivisatos, A. P. Nature 1994, 370, 354-356. (d) Holmes, J. D.; Johnston, K. P.; Doty, R. C.; Korgel, B. A. Science 2000, 287, 14711473. (e) Hiruma, K.; Yazawa, M.; Katsuyama, T.; Ogawa, K.; Haraguchi, K.; Koguchi, M.; Kakibayashi, H. J. Appl. Phys. 1995, 77, 447-462. (f) Yang, J.; Meldrum, F. C.; Fendler, J. H. J. Phys. Chem. 1995, 99, 55005504. (g) Chen, C.-C.; Chao, C.-Y.; Lang, Z.-H. Chem. Mater. 2000, 12, 1516-1518.

(3) (a) Ponce, F. A.; Bour, D. P. Nature 1997, 386, 351-359. (b) Morkoc, H.; Mohammad, S. N. Science 1995, 267, 51-55. (c) Nakamura, S. Science
} 1998, 281, 956-961. reaction $^{5}$, and catalytic reaction. ${ }^{6}$ In particular, a large number of crystalline $\mathrm{GaN}$ nanowires have been obtained by a catalytic synthesis based on a vapor-liquid-solid (VLS) growth mechanism; ${ }^{4,6}$ however, a systematic study of the growth and characterization of such high-quality GaN nanowires that allowed reliable testing of their optoelectronic properties was still lacking.

Recently, we reported a large-scale synthesis of GaN nanowires from the catalytic reaction of gallium and ammonium using indium powder as a catalyst. ${ }^{6}$ In our initial synthesis, the purity and diameters of the nanowires were still not well-controlled. Subsequently, breakthroughs have been made to achieve highpurity and -quality $\mathrm{GaN}$ nanowires. In this work, new experimental parameters were applied to carry out a step-by-step optimization of the nanowire growth based on our previous results. First, the experimental setup was modified to separate residual gallium and bulk gallium nitride from resulting $\mathrm{GaN}$ nanowires after the catalytic reaction. Second, besides indium powder, various types of metals were introduced into the reaction to serve as a catalyst for nanowire growth. Third, the experimental parameters were carefully adjusted, including the reaction temperatures, time, and heating rates to increase the yield of the nanowires and, furthermore, to control their diameters. These systematic optimizations have led to significant progress in preparing high-purity and -quality GaN nanowires. Thus, further investigations of their optical properties and potential applications became feasible.

A study of the optical properties of GaN nanowires was desirable, because it directly links to their potential optoelec-

(4) Duan, X. F.; Lieber, C. M. J. Am. Chem. Soc. 2000, 122, 188-189.

(5) Han, W.; Fan, S.; Li, Q.; Hu, Y. Science 1997, 277, 1287-1289.

(6) Chen, C.-C.; Yeh, C.-C. Adv. Mater. 2000, 12, 738-741. 
tronic applications. Indeed, it has already been established that the effects of quantum confinement in InN clusters of the InGaN active layer were responsible for the high brightness of the lightemitting diodes. ${ }^{7}$ It was based on the argument that once captured by quantum clusters, carriers occupy strongly localized states, and their migration toward nonradiative recombination centers were hindered. Therefore, it would be quite interesting to know whether efficient luminescent properties can be achieved in GaN quantum wires. Furthermore, the growth of GaN nanowires for optical luminescence applications on silicon substrate were highly desirable due to the mature development and large-scale production of $\mathrm{Si}$ substrates at low cost and considerable size.

To provide additional information on the optical properties of $\mathrm{GaN}$ nanowires, we used the Raman-scattering technique to investigate the vibrational properties of the material. Ramanscattering has been proved to be a valuable tool for probing phonon excitations in semiconductors. ${ }^{8}$ Recently, it has also been applied to the characterization of nano-structured semiconductors. ${ }^{9}$ Of principal interest is Raman-scattering evidence for the changes of both the phonon structure and the electron-phonon coupling of the systems due to quantum confinement or synthesis-specific structural modification. Indeed, it has been shown in this study that a strong correlation between the Raman phononic excitation spectra and the finite-size effects in $\mathrm{GaN}$ nanowires exists.

The nanosized wire-like structures grown on a large-scale surface are ideal for the field emission applications such as field emission display (FED). Recently, the potential uses of carbon nanotubes in FED have been reported in the literature. ${ }^{10}$ The extremely large enhancement factor due to their high aspect ratios is thought to be the key to the low turn-on field and high emission current; however, poor emission stability of carbon nanotubes, which may be due to the progressive destruction of carbon nanotubes under such a high emission current, has also been addressed. ${ }^{11}$ In comparison to carbon nanotubes, semiconductor nanowires or nanorods possess similar geometric features but more rigid structures. It has been reported that $\mathrm{Si}$ and $\mathrm{SiC}$ nanowires ${ }^{12}$ as well as $\mathrm{SiCN}$ nanorods ${ }^{13}$ exhibit promising field emission properties. Because the diameters of $\mathrm{GaN}$ nanowires reported in this paper were $<10 \mathrm{~nm}$, good field emission properties due to the high enhancement factor and stable structure were expected.

(7) (a) Naxamura, S.; Fasol, G. The Blue Laser Diode; Springer-Verlag: Berlin, 1997. (b) Gerard, J. M.; Cabrol, O.; Sermage, B. Appl. Phys. Lett. 1996, 68, 3123-3125. (c) Yang, H. C.; Kuo, P. F.; Lin, T. Y.; Chen, Y. F. Appl. Phys. Lett. 2000, 76, 3712-3714.

(8) Cardona, M. Light Scattering in Solid; Springer-Verlag: Berlin, 1975.

(9) (a) Li, G. H.; Ding, K.; Chen, Y.; Han, H. X.; Wang, Z. P. J. Appl. Phys. 2000, 88, 1439-1442 and references therein. (b) Teo, K. L.; Kwok S. H.; Yu, P. Y.; Guha, S. Phys. Rev. B 2000, 62, 1584-1587 and references therein. (c) Nanda, K. K.; Sahu, S. N.; Soni, R. K.; Tripathy, S. Phys. Rev. $B$ 1998, 58, 15405-15407 and references therein.

(10) (a) Ren, Z. F.; Huang, Z. P.; Xu, J. W.; Wang, J. H.; Bush, P.; Siegal, M. P.; Provencio, P. N. Science 1998, 282, 1105-1107. (b) Choi, W. B.; Chung, D. S.; Kang, J. H.; Kim, H. Y.; Jin, Y. W.; Han, I. T.; Lee, Y. H.; Jung, J. E.; Lee, N. S.; Park, G. S.; Kim, J. M. Appl. Phys. Lett. 1999, 75, 3129-3131.

(11) Bonard, J.-M.; Salvetaat, J.-P.; Stockli, T. W.; de Heer, W. A.; Forro, L.; Chatelain, A. Appl. Phys. Lett. 1998, 73, 918-920.

(12) (a) Au, F. C. K.; Wong, K. W.; Tang, Y. H.; Zhang, Y. F.; Bello, I.; Lee, S. T. Appl. Phys. Lett. 1999, 75, 1700-1702. (b) Pan, Z.; Lai, H.L.; Au, F. C. K.; Duan, X.; Zhou, W.; Shi, W.; Wang, N.; Lee, C.-S.; Wong, N.-B.; Lee, S.-T.; Xie, S. Adv. Mater. 2000, 12, 1186-1190.

(13) (a) Chen, K. H.; Wu, J. J.; Chen, L. C.; Wen, C. Y.; Kichambare, P. D.; Tarntair, F. G.; Kuo, P. F.; Chang, S. W.; Chen, Y.-F. Diamond Relat. Mater. 2000, 9, 1249-1256. (b) Tarntair, F. G.; Wen, C. Y.; Chen, L. C.; Wu, J. J.; Chen, K. H.; Kuo, P. F.; Chang, S. W.; Chen, Y. F.; Hong, W. K.; Cheng, H. C. Appl. Phys. Lett. 2000, 76, 2630-2632.

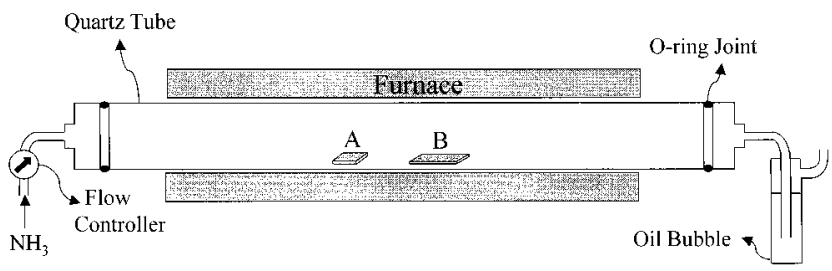

Figure 1. Schematic experimental setup for the growth of $\mathrm{GaN}$ nanowires. Gallium and catalyst were placed on substrates A and B, respectively. Typical experimental condition: iron powder used as catalyst on silicon substrate; heating rate, $50{ }^{\circ} \mathrm{C} / \mathrm{min}$; reaction temperatures, $910^{\circ} \mathrm{C}$; and total reaction time, $12 \mathrm{~h}$ under an ammonium flow of $18 \mathrm{sccm}$.

\section{Experimental Section}

Materials and Reagents. Substrates of silicon (100) wafer or quartz plates were cleaned by a standard treatment in piranha solution $(30 \%$ $\mathrm{H}_{2} \mathrm{O}_{2} / 70 \% \mathrm{H}_{2} \mathrm{SO}_{4}$ ) and rinsed in deionized water before use. Molten gallium $(99.9999 \%)$ and catalysts including the metal powder (1-10 $\mu \mathrm{m}$ in particle size) of indium $(99.9999 \%)$, iron (99.5\%), nickel $(99.8 \%)$, and cobalt $(99.99 \%)$, and the metal complexes of ferrocene $\left(\mathrm{C}_{10} \mathrm{H}_{10} \mathrm{Fe}, 98 \%\right)$, iron phthalocyanine $\left(\mathrm{C}_{32} \mathrm{H}_{16} \mathrm{~N}_{8} \mathrm{Fe}, 96 \%\right)$, cobalt phthalocyanine $\left(\mathrm{C}_{32} \mathrm{H}_{16} \mathrm{~N}_{8} \mathrm{Co}, 96 \%\right)$, and nickel phthalocyanine $\left(\mathrm{C}_{32} \mathrm{H}_{16} \mathrm{~N}_{8^{-}}\right.$ $\mathrm{Ni}, 95 \%)$ were used as received. The metal alloys were obtained from the mixtures of metal nitrate solutions. Toluene was purified by distillation before use. Ammonium gas was purged through a gas purifier to remove water and oxygen before the reaction.

Catalyst Preparation. The catalysts were placed on substrate B (see below) in different ways: (1) Indium, iron, nickel, and cobalt powders were suspended in toluene by sonication and then homogeneously dissipated onto the substrate. (2) Ferrocene, iron phthalocyanine, cobalt phthalocyanine, nickel phthalocyanine, and metal nitrates were dissolved in toluene or water to form a solution. The solution was then dispersed onto the substrate and dried by a flow of nitrogen. The substrates were eventually dried in a tube furnace to remove all remaining solvent before the reaction. (3) For bimetallic alloy catalysts, any two of the metal $(\mathrm{Ni}, \mathrm{Co}, \mathrm{Fe})$ nitrates with 1:1 molar ratio were dissolved in water to form a solution. The solution was dispersed onto the substrate and dried as described above. The metal alloys were formed while the substrate was heated to high temperatures.

Growth of Gallium Nitride Nanowires. The experimental setup for GaN nanowire growth is described schematically in Figure 1. Typically, an excess amount of molten gallium was placed on substrate $\mathrm{A}\left(1 \times 1 \mathrm{~cm}^{2}\right)$ that was inserted into the center of a 1-in.-diameter quartz tube placed inside a tube furnace. Substrate B $\left(2 \times 2 \mathrm{~cm}^{2}\right)$ deposited with catalysts $(1-5 \mathrm{mg})$ was transferred into the quartz tube at a distance $1-10 \mathrm{~cm}$ away from substrate $\mathrm{A}$. The quartz tube was degassed and then purged with ammonia. The temperature of the furnace was increased at a rate of $30-100{ }^{\circ} \mathrm{C} / \mathrm{min}$ from room temperature to the reaction temperature of $800-1050{ }^{\circ} \mathrm{C}$ and kept for $3-48 \mathrm{~h}$ under a constant flow of ammonia $(18 \mathrm{sccm})$. After the reaction, the furnace was cooled to room temperature and the resulting nanowires were collected on the surface of substrate B.

Size, Morphology, and Structure Characterization. The morphologies and crystal structures of the resulting material on substrate $B$ were characterized using scanning electron microscopy (SEM, JEOLJSM-6300) and X-ray powder diffraction (XRD, Toshiba, A-40-Cu). Further structure and stoichiometry analyses of individual GaN nanowires were performed using transmission electron microscopy (TEM, Zeiss-10C at $100 \mathrm{kV}$ ), high-resolution TEM (HRTEM, JEOL-4000 EX at $400 \mathrm{kV}$ ), selected-area electron diffraction, and energy-dispersive $\mathrm{X}$-ray spectroscopy (EDX). The TEM samples were prepared by depositing an aliquot of $\mathrm{GaN}$ nanowires dispersed in toluene onto either an amorphous carbon film or a porous carbon film on a $\mathrm{Cu}$ grid.

Optical Spectroscopy. (1) Photoluminescence Spectra: A chopped $\mathrm{He}-\mathrm{Cd}$ laser working at $325 \mathrm{~nm}$ with an output power of $\sim 10 \mathrm{~mW}$ was used as an excitation source. The sample was placed in a closedcycle He cryostat, which provides a temperature range from 10 to 300 $\mathrm{K}$. The emission signal was collected by a SPEX $0.85-\mathrm{m}$ double 


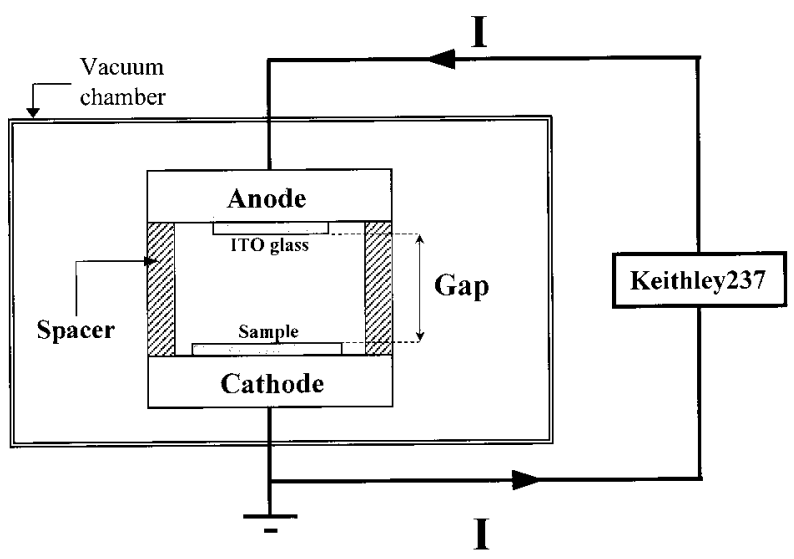

Figure 2. Schematic illustration of the field emission measurement setup. Indium tin oxide (ITO) glass was used as the anode to monitor the emission current.

spectrometer and detected by a lock-in amplifier coupled with a photomultiplier tube.

(2) Raman Spectra: The room-temperature Raman spectra were measured with $514.5 \mathrm{~nm}$ photons from an $\mathrm{Ar}^{+}$laser (Spectra Physics Model 2017). The linearly-polarized light was focused onto the sample through a $100 \times$ optical microscope objective $(0.95 \mathrm{~N}$. A.) having a spatial resolution of $<2 \mu \mathrm{m}$ in a backscattering geometry. Several locations of each sample were probed to ensure reproducibility of the data. The laser power used was $<1.5 \mathrm{~mW}$. The possible effect of local heating was checked by varying the excitation power, and no appreciable change in the Raman spectra was observed for powers $\lesssim 5$ $\mathrm{mW}$. The scattered light without polarization analysis was collected and dispersed by a Dilor XY 800 triple spectrometer having 1800 grooves/mm grating. A liquid-nitrogen-cooled 1024-pixel-wide chargecoupled detector (Jobin Yvon model Spectrum One) was used to record the entire spectrum simultaneously, providing a multiplexing advantage. The spectral resolution of the system was typically $<1 \mathrm{~cm}^{-1}$. To investigate further the resonance property, both $\mathrm{He}-\mathrm{Ne}(632.8 \mathrm{~nm})$ and $\mathrm{Ar}^{+}$lasers $(457.9 \mathrm{~nm}$ ) were used for the study. Detailed Raman analysis using the two excitation lines will be published elsewhere. ${ }^{14}$

Field Emission Measurements. Field emission characteristics were carried out using a parallel-plate configuration of $\mathrm{I}-\mathrm{V}$ measurement under a base pressure of $1 \times 10^{-7}$ Torr at room temperature. A schematic diagram of the field emission measurement setup is shown in Figure 2. A Keithely 237 electrometer was employed for supplying the voltage and measuring the current. The measurement was performed by collecting electrons emitted from the sample while applying a positive voltage on an indium-tin-oxide-coated (ITO) glass electrode, which was placed $70 \mu \mathrm{m}$ above the sample. The area of the anode was restricted to being smaller than that of the sample to avoid the field emission at the sample edges due to their structure enhancement, which would lead to false analyses of field emission characteristics of the $\mathrm{GaN}$ nanowires. Precaution is required to clean the sample before measurement in order to eliminate dust particles on the surface that would also cause false emission current signals.

\section{Results and Discussion}

Systematic Optimization of GaN Nanowire Growth. (1) Varying the Distance between Substrates A and B. Some modifications from the previous setup ${ }^{6}$ were made to improve the purity and diameter distribution of resulting $\mathrm{GaN}$ nanowires. As described in Figure 1, substrates A and B were separated by a distance of $1-10 \mathrm{~cm}$ carrying gallium and catalyst, respectively. After the reaction, it was found that residual gallium and gallium nitride crystals were found only on substrate A, and they were successfully separated from high purity GaN nanowires formed on substrate B. The surface of substrate B exhibited a light yellow, otherwise transparent, color and was

(14) Liu, H. L. et al. Appl. Phys. Lett., submitted.
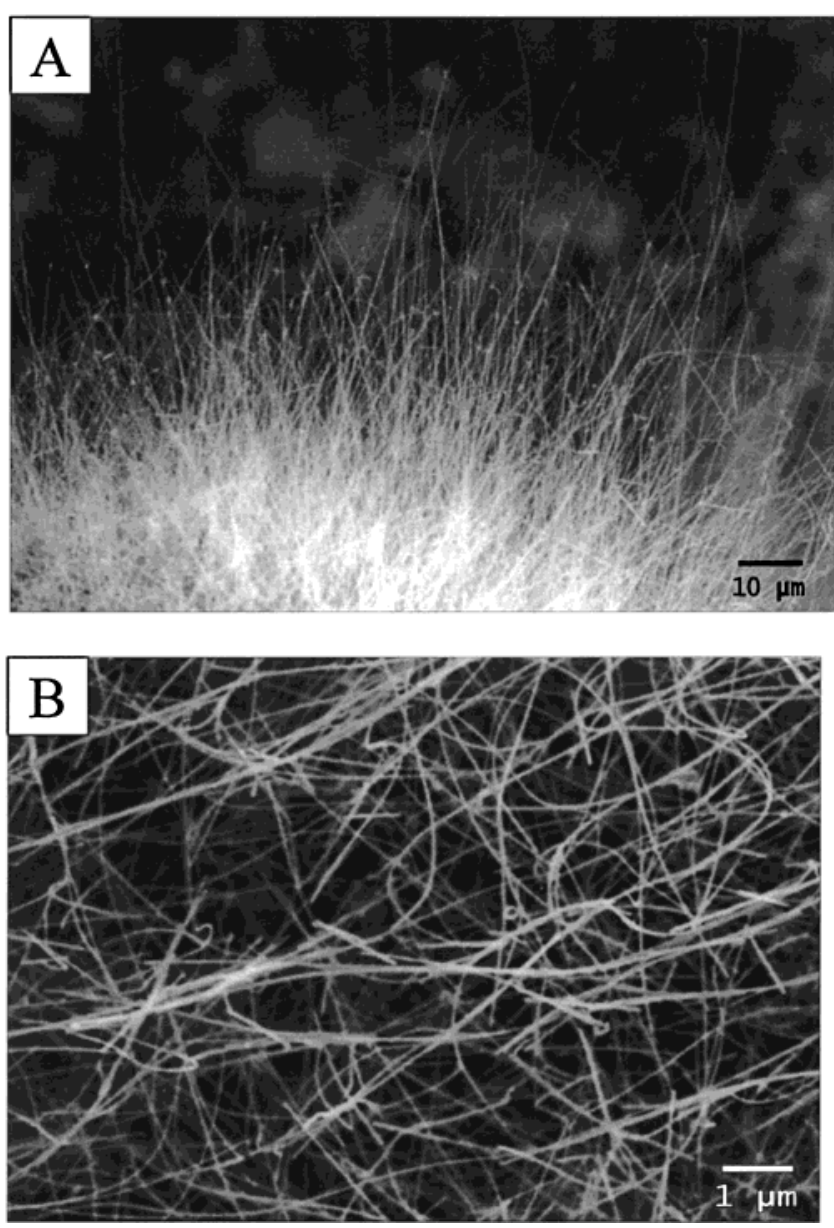

Figure 3. Typical SEM images of GaN nanowires grown on a silicon substrate. (a) Cross-sectional image showing a large number of nanowires grown on substrate B. (b) Top-view image of nanowires. The lengths of the nanowires here are up to several micrometers, and their diameters are $\sim 20-50 \mathrm{~nm}$. The nanowires show a smooth surface and no ramification over their length.

covered entirely by the nanowires without any irregular-shaped crystals. Figure 3 shows typical SEM images of GaN nanowires deposited on substrate B. Further structure and stoichiometry analyses below confirm that those wire-like materials in the SEM images are indeed composed of GaN crystals.

It was found that the diameter distribution of the nanowires collected on substrate B was strongly dependent on the distances between the two substrates. When substrate B was placed closer to substrate $\mathrm{A}$, more nanowires ${ }^{15}$ with larger diameters were obtained. For instance, the diameters of the nanowires were mostly in the range of $50-150 \mathrm{~nm}$ when the substrate B was placed at a distance of $1 \mathrm{~cm}$ from substrate A. In comparison, the diameters of the nanowires were significantly reduced to 20-50 $\mathrm{nm}$ when the distance between the two substrates was $10 \mathrm{~cm}$

(2) Varying the Catalytic Materials. It was reported in our previous study ${ }^{6}$ that indium metal can serve as a good catalyst in the reaction of gallium and ammonium for the growth of $\mathrm{GaN}$ nanowires. In this work, a series of metal powders, metal alloys, and metal complexes were systematically tested in the

(15) Because there are some difficulties in calculating the quantitative yield of the nanowires, relative coverage fractions in volume of the nanowires on substrate B $\left(2 \times 2 \mathrm{~cm}^{2}\right)$ were compared in different samples on the basis of their SEM images. Here, we defined the samples with high yield as that substrate B surface was covered by nanowires without any irregularly shaped crystals. 


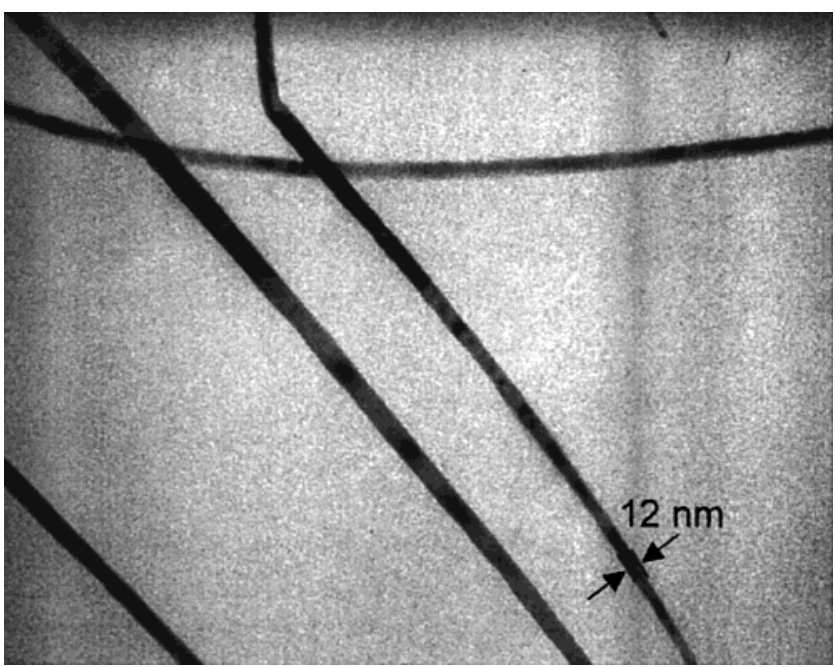

Figure 4. TEM image of GaN nanowires prepared from the catalytic reaction when metal complex of cobalt phthalocyanine was used as a catalyst. The diameters of the nanowires are $<20 \mathrm{~nm}$, as indicated in the image. This image shows some bright and dark contrast bands along the growth direction. These bands may arise from some bending of the nanowires.

catalytic reaction. We found that metal powders and alloys of In, $\mathrm{Co}, \mathrm{Ni}, \mathrm{Fe}, \mathrm{Ni} / \mathrm{Co}$, and $\mathrm{Fe} / \mathrm{Co}$ can all be used as catalysts to produce a large amount of $\mathrm{GaN}$ nanowires. The morphologies of the nanowires were similar to those shown in Figure 3. Typically, the diameters of $\mathrm{GaN}$ nanowires were in the range of $20-50 \mathrm{~nm}$, and their lengths were up to several $\mu \mathrm{m}$, as shown in Figure 3. However, diameters and lengths of GaN nanowires was found to be independent of the metal powders and alloys that were used.

The metal complexes of ferrocene, iron phthalocyanine, cobalt phthalocyanine and nickel phthalocyanine were also used as the catalyst in the reaction of gallium and ammonium for the growth of GaN nanowires. After the catalytic reaction, a large number of $\mathrm{GaN}$ nanowires were obtained, and their morphologies were similar to those shown in Figure 3. However, significant reduction in the average diameters of $\mathrm{GaN}$ nanowires was observed, in particular, for the samples when cobalt phthalocyanine or nickel phthalocyanine was used as a catalyst. A minimum diameter of $6 \mathrm{~nm}$ has been achieved. Figure 4 shows the bright-field TEM image of several segments of crystalline GaN nanowires having diameters down to $12 \mathrm{~nm}$ (also see Figure 9 below). These results indicate that using metal complexes instead of metal powders may result in smaller diameters of $\mathrm{GaN}$ nanowires, because these metal complexes may decompose at high temperatures into gaseous species and form very small metal clusters which can serve as a catalyst. Under a typical reaction condition, the catalysts cobalt phthalocyanine and nickel phthalocyanine resulted in GaN nanowire samples having diameters mostly in the range of $10-50 \mathrm{~nm}$, whereas other powder-type catalysts in our experiments produced nanowires having diameters generally in the range of 20$100 \mathrm{~nm}$.

(3) Varying the Heating Rates. Various heating rates (25, 50,75 , and $>100{ }^{\circ} \mathrm{C} / \mathrm{min}$ ) were tested in the catalytic reaction. When metal powders of $\mathrm{Fe}$, $\mathrm{Co}$, and $\mathrm{Ni}$ were applied in the reaction, $\mathrm{GaN}$ nanowires with a broad diameter distribution were produced under fast heating rates $\left(>100{ }^{\circ} \mathrm{C} / \mathrm{min}\right)$, whereas nanowires with a narrow diameter distribution were produced under slow heating rates $\left(25^{\circ} \mathrm{C} / \mathrm{min}\right.$.). In contrast, when metal

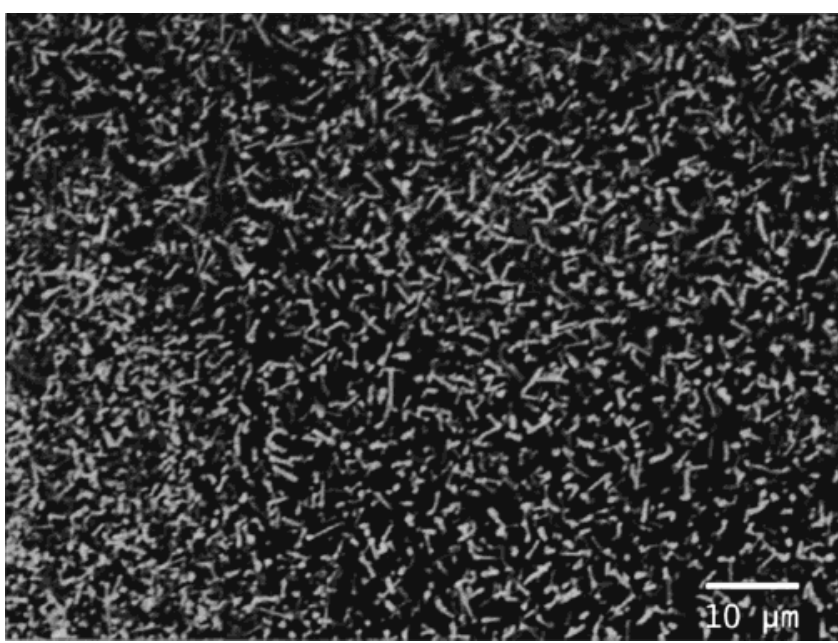

Figure 5. SEM image of the sample heated for $3 \mathrm{~h}$. A large number of short rodlike structures with diameters of several hundred $\mathrm{nm}$ are found.

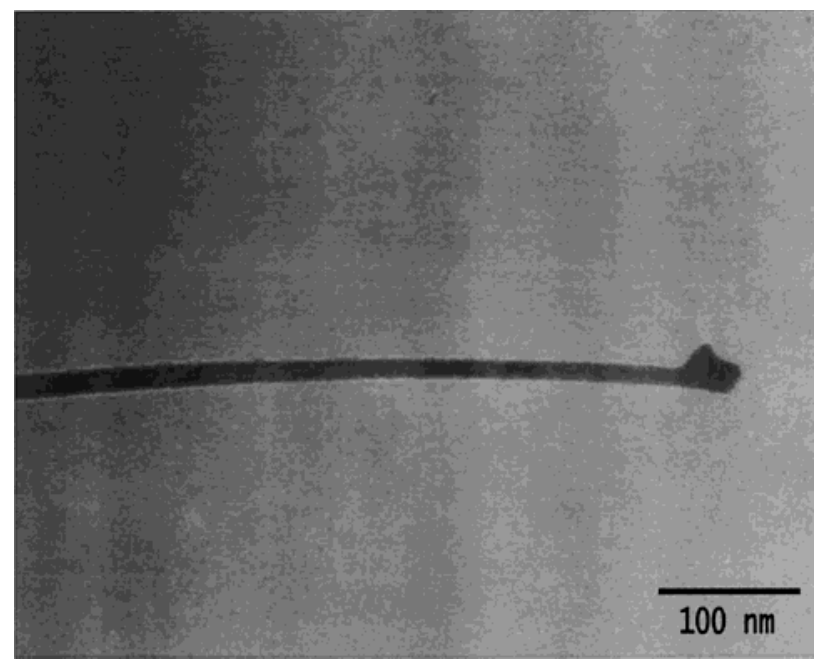

Figure 6. TEM image of a single GaN nanowire. An irregular-shaped nanoparticle is terminated on the tip of the nanowire. The nanowire was prepared using cobalt phthalocyanine as a catalyst. The diameter of the nanowire is $\sim 16 \mathrm{~nm}$.

complexes of $\mathrm{Fe}, \mathrm{Co}$, and $\mathrm{Ni}$ were applied, a slow heating rate led to a broad distribution of diameters of the nanowires and vice versa.

(4) Varying the Total Reaction Time. The total reaction time was a critical factor for determining the diameters and lengths of resulting $\mathrm{GaN}$ nanowires. The catalytic reactions were performed for $3,6,12,18,24$, and $48 \mathrm{~h}$. Figure 5 shows the SEM image of the materials synthesized under a reaction time of $3 \mathrm{~h}$. The material in this image exhibited short rodlike structures. It was found that, during the heating period of 3-12 $\mathrm{h}$, these rodlike structures continuously grew in length along the axial direction to form wire-like structures, whereas their diameters decreased. These results agreed with the VLS growth mechanism of nanowires in that the growth was initiated from a saturated miscible liquid alloy of $\mathrm{Ga}-\mathrm{N}-$ catalyst, and then $\mathrm{GaN}$ nanowires were precipitated out in one direction as described below (see Figure 7). However, when the reaction time was more than $12 \mathrm{~h}$, the diameters of nanowires were dramatically increased. Unusual shapes of GaN bulk crystals were observed when the total reaction time exceeded $48 \mathrm{~h}$.

(5) Varying the Reaction Temperatures. The optimal reaction temperature for the formation of $\mathrm{GaN}$ nanowires was 
(a)

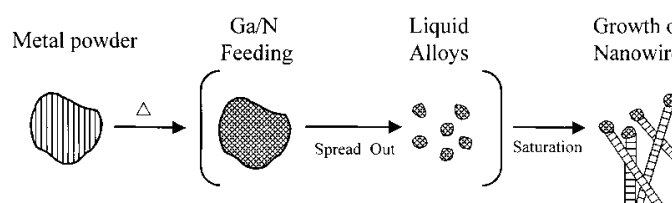

(b)

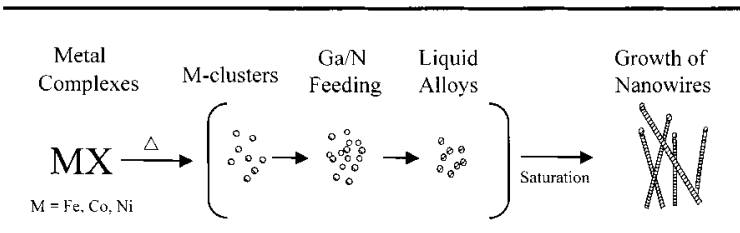

Figure 7. Schematic illustration of two possible pathways of the VLS growth of GaN nanowires. (a) At the beginning, the catalysts of metal powder ( $>1 \mu \mathrm{m}$ in diameter) gradually form catalyst - gallium - nitrogen alloys after gallium vapor transport to the catalysts on substrate B. Each alloy turns into a miscible liquid droplet and then spreads out, becoming many smaller droplets. Hence, after the concentration of gallium nitride reaches saturation in the droplet, the droplet can act as a nucleation site, and a GaN nanowire begins to grow in one direction. (b) At the initial heating stage, metal complexes are decomposed rapidly in the gas phase or on the substrate and sequentially generate many small metal clusters. These clusters form small $\mathrm{Ga}-\mathrm{N}-$ catalyst liquid droplets and serve as nucleation sites after gallium and ammonium vapors are fed in. In comparison to pathway (a), the nanowires with smaller diameters could be obtained when the reaction goes through pathway (b).

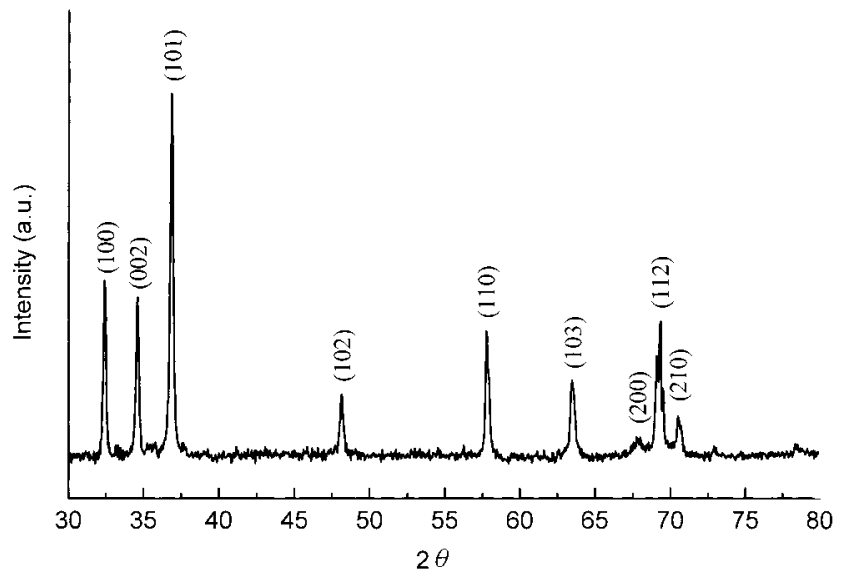

Figure 8. X-ray powder diffraction of $\mathrm{GaN}$ nanowires. The sample was prepared under the conditions as described in the caption of Figure 1.

found to be $\sim 910{ }^{\circ} \mathrm{C}$. When the reaction temperature was $<$ $850^{\circ} \mathrm{C}$, the crystallinity of the resulting materials became poor and an amorphous solid was obtained. When the reaction temperatures were $>1050{ }^{\circ} \mathrm{C}$, almost no $\mathrm{GaN}$ nanowires on substrate B were observed.

Overall, following the systematic optimizations of experimental conditions, significant progress was made toward controlling the growth of GaN nanowires. High-purity and -quality GaN nanowires with diameters in the range of 10-50 $\mathrm{nm}$ were available for further studies of their structural, optical, and electrical properties for further applications.

Vapor-Liquid-Solid (VLS) Growth of GaN Nanowires. A typical TEM image of a single nanowire is displayed in Figure 6 to illustrate the details of the nanowire growth. In this image, the nanowire was terminated with a nanoparticle at its tip. The EDX analyses indicated that the nanoparticle on the tip was mainly the catalytic metal, gallium, and nitrogen, whereas the nanowire was only composed of gallium and nitrogen. The result provides a clear evidence that the growth of nanowires went through a VLS process. Similar phenomena have been commonly observed in the VLS growth of some ceramic whiskers such as $\mathrm{AlN}, \mathrm{SiC}$, and TaC. ${ }^{16}$

Two possible pathways were proposed and described schematically in Figure 7 to further illustrate the details of the VLS growth of GaN nanowires. On the basis of the proposed pathways and the experimental results, we summarized several important factors for preparing high-purity and -quality nanowires using the catalytic reaction. (1) The formation of miscible liquid alloys of $\mathrm{Ga}-\mathrm{N}-$ catalyst at certain reaction temperatures ( $\sim 910^{\circ} \mathrm{C}$ in this experiment) is an essential step to induce the VLS growth of the nanowires. Our experimental results have demonstrated that several different metals can serve as good catalysts, suggesting that those metal catalysts must be miscible with $\mathrm{Ga}$ and $\mathrm{N}$ to form liquid alloys as described in Figure 7. (2) The diameters of resulting $\mathrm{GaN}$ nanowires are dependent on the sizes of the miscible liquid alloys of $\mathrm{Ga}-\mathrm{N}-$ catalyst, provided that the miscible liquid alloys were well-dispersed. ${ }^{17}$ Using metal complexes as a catalyst can result in $\mathrm{GaN}$ nanowires with very small diameters, suggesting that gas-phase condensation of the metal complexes produced very small miscible liquid alloys. Meanwhile, the initial heating rates may also affect the sizes of the miscible liquid alloys and consequently change the diameters of the nanowires as well as their distribution. (3) According to the VLS processes shown in Figure 7, gallium vapor must have first evaporated and transported from substrate A to substrate $\mathrm{B}$, where the catalytic reaction has taken place, therefore, high purity $\mathrm{GaN}$ nanowires can be separated from residual gallium metal. The average diameters of the nanowires varied with the distances between substrates A and B due to the changes of the concentrations of gallium vapor.

Besides the factors discussed above, it is believed that other parameters may also play a role on the VLS growth of GaN nanowires. More efforts should be made to obtain GaN nanowires with a well-dispersed aspect ratio (diameter/length) soon.

Structural Characterization. A typical XRD pattern of the resulting GaN nanowires is shown in Figure 8. The sharp diffraction peaks in the pattern were indexed to a hexagonal wurtzite structure. The lattice constants derived from the peak positions were $a=3.188$ and $c=5.180 \AA$, which agreed well with the reported values of bulk GaN crystals. ${ }^{18}$ The strong diffraction peaks relative to the background signals suggested that the resulting $\mathrm{GaN}$ nanowires had a high purity of the $\mathrm{GaN}$ wurtzite phase. We have found that almost all nanowire samples had growth directions of [110] or [100] in parallel to the long axis of the nanowires, ${ }^{6}$ as shown from the HRTEM investigations, however, it should be mentioned that the nanowires obtained from other reports have a common growth direction of [100]. , $^{4,5}$

As can be seen from HRTEM image in Figure 9, the resulting nanowires have diameters $<10 \mathrm{~nm}$ when metal complexes were applied as catalysts. No defects between contrast bands were found in the HRTEM image. The clear lattice fringes in this image confirmed a single-crystal structure of the nanowires, as is evident from the clear lattice fringes in this image. Also noted

(16) (a) Haber, J. A.; Gibbons, P. C.; Buhro, W. E. Chem. Mater. 1998 , 10, 4062-4071. (b) Chrysanthou, A.; Grieveson, P.; Jha, A. J. Mater. Sci. 1991, 26, 3463-3476. (c) John, M.; Nygren, M. J. Mater. Res. 1997, 12, 2419-2427.

(17) (a) Gudiksen, M.; Lieber, C. M. J. Am. Chem. Soc. 2000, 122, 88018802. (b) Duan, X.; Lieber, C. M. Adv. Mater. 2000, 12, 298-301.

(18) (a) Perlin, P.; Carillon, C. J.; Itie, J. P.; Miguel, A. S. Phys. Rev. B 1992, 45, 83-89. (b) Lagerstedt, O.; Monemar, B. Phys. Rev. B 1979, 19, 3064-3070. 


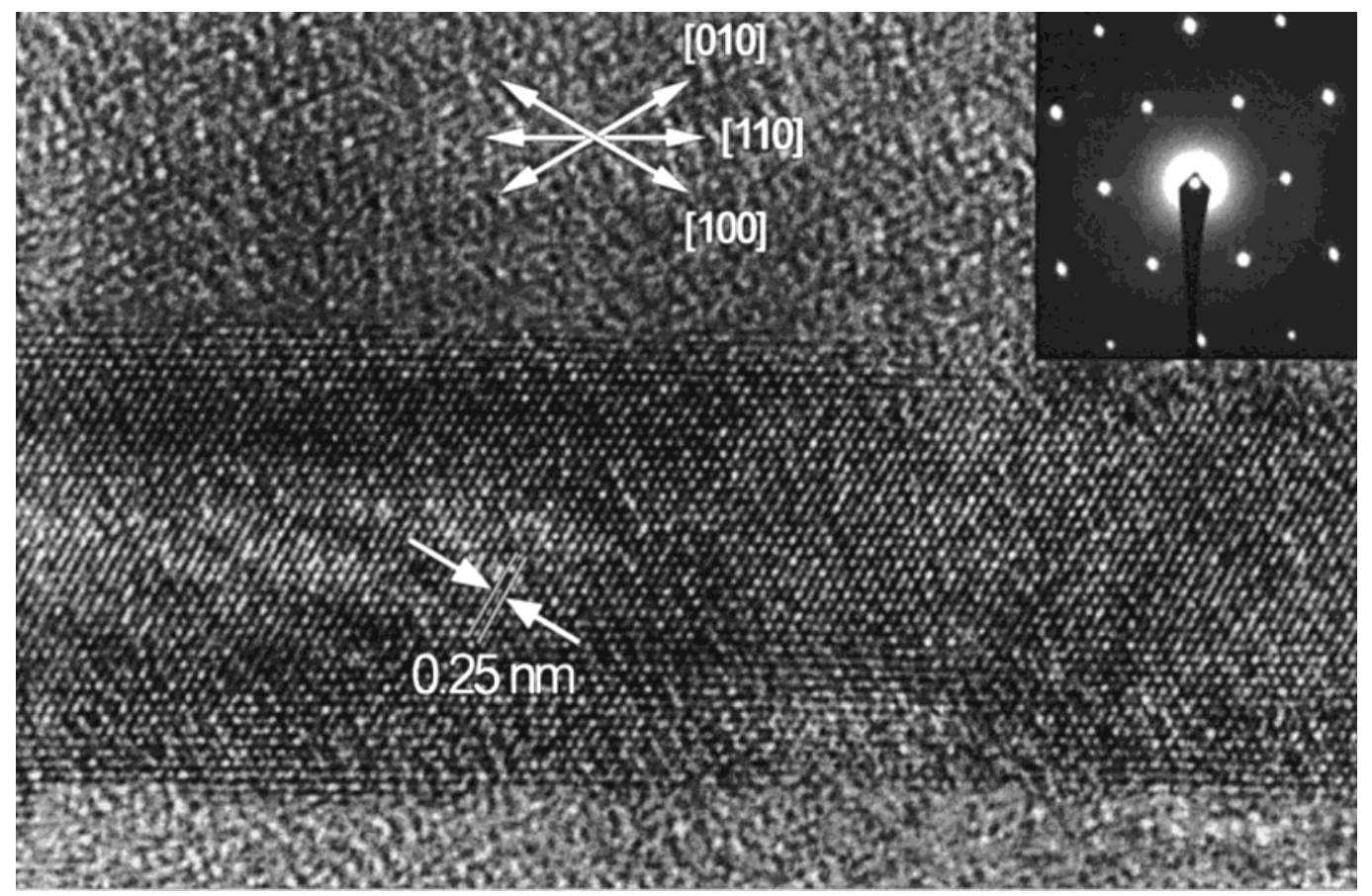

Figure 9. Typical high-resolution TEM image and its corresponding electron diffraction (inset) of a nearly defect-free GaN nanowire prepared using cobalt phthalocyanine as a catalyst. The direction of the long axis is [110], and the zone axis is [001]. The diameter of this single nanowire is $\sim 8 \mathrm{~nm}$. The space of $\sim 0.25 \mathrm{~nm}$ between arrowheads corresponds to the distance between two (100) planes.

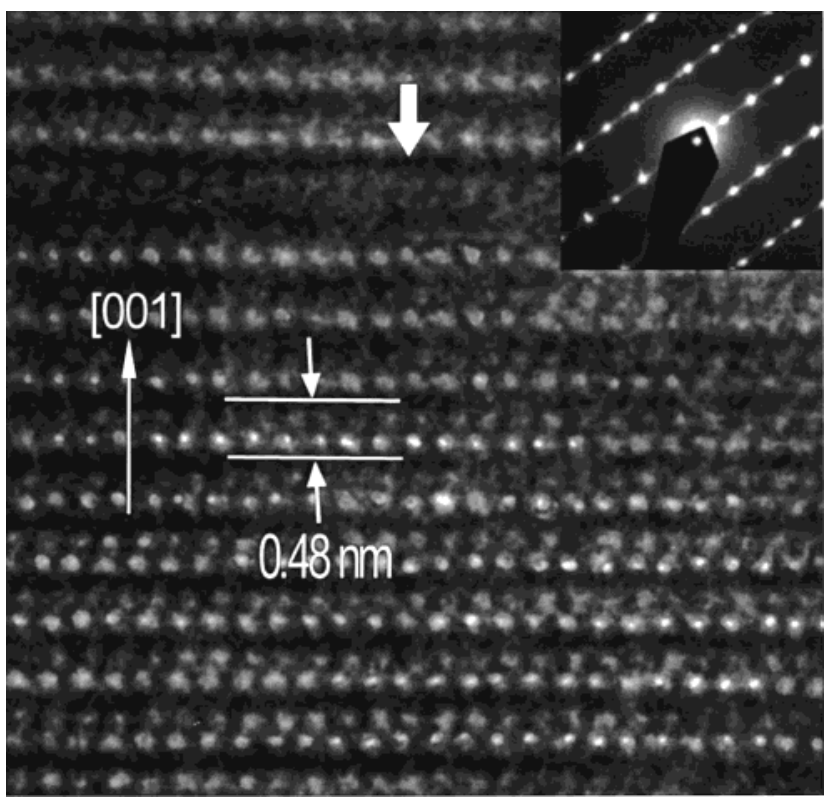

Figure 10. High-resolution TEM image and its corresponding electron diffraction (inset) of a faulted $\mathrm{GaN}$ nanowire prepared using iron powder as the catalyst. The direction of the long axis is [001], and the zone axis is [100]. The faulted structure is marked by an arrowhead.

in this image is that the [110] direction was parallel to the long axis of the wires, indicating that [110] direction is the fastgrowth direction for $\mathrm{GaN}$ nanowires. The inset of Figure 9 shows a selected-area electron diffraction pattern of the nanowire that can be indexed to the reflection of hexagonal GaN crystals along [001] directions.

Figure 10 shows a high-resolution TEM image of a GaN nanowire observed from the [100] zone axis, and the inset shows the corresponding electron diffraction pattern. For this specific nanowire, the direction of the long axis is [001]. Along this direction, "stacking faults" were observed in some regions that exhibited a zinc-blend-type structure, of which the [111]

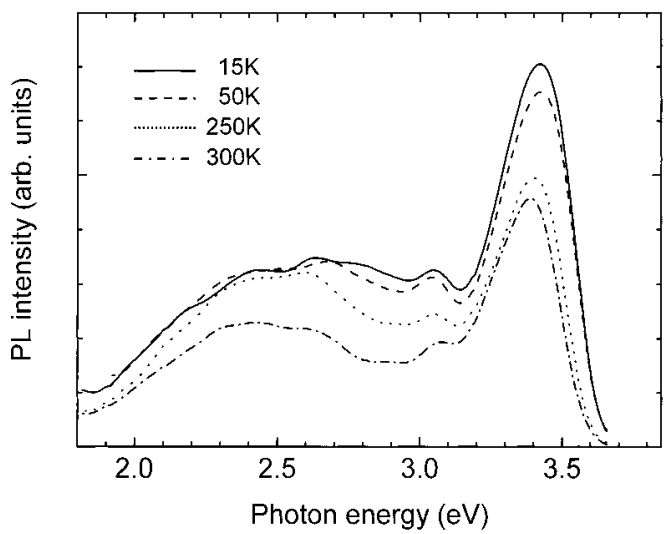

Figure 11. Temperature dependence of photoluminescence spectra of $\mathrm{GaN}$ nanowires. A $\mathrm{He}-\mathrm{Cd}$ laser $(325 \mathrm{~nm})$ with an output power of $>10 \mathrm{~mW}$ was used as the excitation source. Iron powder was used as the catalyst for this sample.

direction was parallel to the [001] direction of the wurtzite structure. Notice that the structures with highly faulted structures were found only in individual nanowires with large diameters. The nanowires having small diameters, as shown in Figure 9, are generally defect-free single crystallites. Further evidence of the existence of a zinc-blend structure in a predominant wurtzite structure of the GaN nanowires can be seen from the photoluminescence data as described below.

Photoluminescence Spectroscopy. Typical photoluminescence spectra from $\mathrm{GaN}$ nanowires taken at different temperatures are shown in Figure 11. It is interesting to note that a strong band-edge emission of wurtzite GaN crystal can be observed at room temperature. The band-edge emission intensity at $300 \mathrm{~K}$ is $\sim 60 \%$ of that at $15 \mathrm{~K}$. In comparison to the results obtained at the same energy range of emission from bulk GaN, the $\mathrm{GaN}$ nanowires exhibit the weakest photoluminescence quenching. ${ }^{2 \mathrm{e}, 19}$ This result indicates that thermal quenching of photoluminescence is much reduced in $\mathrm{GaN}$ quantum wires. The broad half-width of the PL spectra probably reflects the 


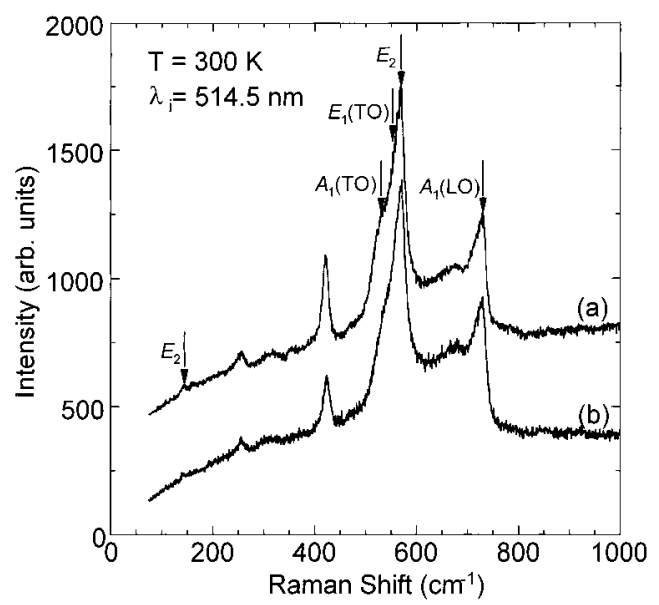

Figure 12. Room-temperature Raman-scattering spectra of two GaN nanowire samples grown by using (a) iron powder and (b) nickel powder as a catalyst.

broad size distribution of GaN nanowires revealed by the SEM pictures as shown in Figure 3. The small shoulder around 3.1 $\mathrm{eV}$ is very close to the band-edge emission of cubic $\mathrm{GaN},{ }^{20}$ in good agreement with the HRTEM result, which indicates the existence of a small fraction of cubic $\mathrm{GaN}$ incorporated in the hexagonal $\mathrm{GaN}$ matrix. A further study of this problem is desirable, which may bring out the necessary information for growing cubic and hexagonal GaN nanowires. Finally, there exists a weak and broad structure centered around $2.3 \mathrm{eV}$ that corresponds to the well-known yellow luminescence observed in bulk GaN. ${ }^{21}$ The weak defect-related signal implies that our GaN nanowires contain few defects and high quality. A more detailed photoluminescence study is in progress and will be published in a separate paper.

Raman Spectroscopy. Figure 12 shows the room-temperature Raman-scattering intensity of two GaN nanowires samples using green $(514.5 \mathrm{~nm})$ excitation. The Raman-scattering response, obtained by dividing the measured spectra by the Bose-Einstein thermal factor, is dominated by a number of optical phonons and an electronic continuum. When the samples were excited with different laser lines ranging from 632.8 to $457.9 \mathrm{~nm}$, the signal for the continuum did not shift for all of the excitations, which suggests that the electronic continuum is, indeed, luminescence. Here, we examine the detailed phononic Raman response of the $\mathrm{GaN}$ nanowires and fit the phonon peaks using a standard Lorentzian profile. Additionally, the broad luminescence background was fitted to a polynomial and subtracted out from the spectrum. The frequencies and assignments of the phonon modes shown in Figure 12 are listed in Table 1. The results for our samples were compared to previous work for GaN nanowires ${ }^{22}$ as well as those observed for bulks. ${ }^{23}$

(19) (a) Lagerstedt, O.; Monemar, B. J. Appl. Phys. 1974, 45, 22662272. (b) Chen, G. D.; Smith, M.; Lin, J. Y.; Jiang, H. X.; Salvador, A.; Sverdlov, B. N.; Botchkarv, A.; Morkoc, H. J. Appl. Phys. 1996, 79, 26752683.

(20) Okumura, H.; Hamaguchi, H.; Feuillet, G.; Ishida, Y.; Yoshida, S. Appl. Phys. Lett. 1998, 72, 3056-3058.

(21) Chen, H. M.; Chen, Y. F.; Lee, M. C.; Feng, M. S. Phys. Rev. B 1997, 56, 6942-6946.

(22) Cheng, G. S.; Zhang, L. D.; Zhu, Y.; Fei, G. T.; Li, L.; Mo, C. M.; Mao, Y. Q. Appl. Phys. Lett. 1999, 75, 2455-2457.

(23) (a) Azuhata, T.; Sota, T.; Suzuki, K.; Nakamura, S. J. Phys.: Condens. Matter 1995, 7, L129-133. (b) Filippidis, L.; Siegle, H. Hoffmann, A.; Thomsen, C.; Karch, K.; Bechstedt, F. Phys. Status Solidi B 1996, 198, 621-627. (c) Davydov, V. Y.; Kitaev, Y. E.; Goncharuk, I. N.; Smirnov, A. N.; Graul, J.; Semchinova, O.; Uffmann, D.; Smirnov, M. B.; Mirgorodsky, A. P.; Evarestov, R. A. Phys. Rev. B 1998, 58, 1289912907.
Table 1. Raman Peaks Observed in the Bulk and Nanowires GaN Spectra at $300 \mathrm{~K}$, and the Corresponding Symmetry Assignments ${ }^{a}$

\begin{tabular}{ccccccl}
\hline $\begin{array}{c}\text { bulk } \\
\mathrm{GaN}^{b}\end{array}$ & $\begin{array}{c}\text { bulk } \\
\mathrm{GaN}^{c}\end{array}$ & $\begin{array}{c}\text { bulk } \\
\mathrm{GaN}^{d}\end{array}$ & $\begin{array}{c}\text { thin } \\
\text { film }\end{array}$ & $\begin{array}{c}\text { nanowires } \\
\mathrm{GaN}^{e}\end{array}$ & $\begin{array}{c}\text { this } \\
\text { work }\end{array}$ & $\begin{array}{c}\text { symmetry } \\
\text { assignment }\end{array}$ \\
\hline 144 & 145 & 144 & 145 & & 144 & $\mathrm{E}_{2}($ low) \\
& & & & & 252 & zone-boundary phonon \\
& & & & & 421 & zone-boundary phonon \\
533 & 533 & 532 & 533 & 531 & 537 & $\mathrm{~A}_{1}(\mathrm{TO})$ \\
561 & 561 & 559 & 561 & 560 & 562 & $\mathrm{E}_{1}(\mathrm{TO})$ \\
569 & 570 & 568 & 571 & & 569 & $\mathrm{E}_{2}($ high) \\
& & & & & 670 & defect-induced \\
& & & & & & phonon mode \\
735 & 735 & 734 & 737 & 733 & 728 & $\mathrm{~A}_{1}(\mathrm{LO})$ \\
743 & 742 & 741 & 743 & 745 & & $\mathrm{E}_{1}(\mathrm{LO})$ \\
\hline
\end{tabular}

${ }^{a}$ Units are in $\mathrm{cm}^{-1}{ }^{b}$ From ref 23a. ${ }^{c}$ From ref 23b. ${ }^{d}$ From ref 23c. ${ }^{e}$ From ref 22 .

Under ambient conditions, GaN crystallizes in the hexagonal wurtzite-type structure (space group $\mathrm{C}_{4 v}^{6}$ ) consisting of two formula units per primitive cell. Because the wavelengths of light used as an excitation source were a small fraction of the brillouin zone, the excitations probed in this study are essentially at $q=0$. According to the factor group analysis, single-crystal $\mathrm{GaN}$ possesses eight sets of optical phonon modes near the zone center. ${ }^{24}$ These modes are classified into Raman $\left(\mathrm{A}_{1}+\mathrm{E}_{1}+\right.$ $\left.2 \mathrm{E}_{2}\right)$, silent $\left(2 \mathrm{~B}_{1}\right)$, and infrared active $\left(\mathrm{A}_{1}+\mathrm{E}_{1}\right)$. Although the $\mathrm{GaN}$ is noncentrosymmetric, the $\mathrm{A}_{1}$ and $\mathrm{E}_{1}$ modes are further split into LO (longitudinal optical) and TO (transverse optical) components. From the results shown in Figure 12 and the fit parameters in Table 1, four interesting phonon properties in our $\mathrm{GaN}$ nanowires have been revealed by light scattering. First, the five observed Raman-active phonon bands near $\sim 144,537$, 562, 569, and $728 \mathrm{~cm}^{-1}$ have symmetries $\mathrm{E}_{2}$ (low), $\mathrm{A}_{1}$ (TO), $\mathrm{E}_{1}$ (TO), $\mathrm{E}_{2}$ (high), and $\mathrm{A}_{1}$ (LO), respectively. The frequencies of the first four modes are close to those previously reported on nanowires ${ }^{22}$ and bulks, ${ }^{23}$ but in our samples, the position of the $A_{1}(L O)$ mode is significantly softer. Second, we find that these phonon peaks broaden substantially in the spectra of the nanowires. The line width increases roughly by a factor of 2 to 5 , as compared to bulk. Third, the strong $\mathrm{E}_{2}$ (high) phonon line in the spectra reflects the characteristics of the hexagonal crystal phase of the GaN nanowires. Fourth, three additional modes are also observed at $\sim 252,421$, and $670 \mathrm{~cm}^{-1}$, all of which are not allowed by the $\mathrm{C}_{4 v}^{6}$ space group in first-order Ramanscattering at the zone center. Possible mechanisms that could bring about these features are discussed below.

Let us focus first on the broadening of the five first-order modes in the Raman spectra of the GaN nanowires. This behavior is in line with the spatial-correlation or phonon confinement model, ${ }^{25}$ which suggests that the phonons in nanometric-sized systems can be confined in space by crystallite boundaries or surface disorders. Consequently, this confinement causes an uncertainty in the wave vector of the phonons, which results in broadening of the Raman features. Similar evidence for a broadening of the Raman bands associated with the phonon confinement effects has been observed in the micro- and nanocrystallite systems, including diamond, ${ }^{26} \mathrm{Si}^{27} \mathrm{Ge},{ }^{28}$ and GaAs. ${ }^{27}$

(24) Cardona, M.; Güntherodt, G. Light Scattering in Solids II; SpringerVerlag: Berlin, 1982.

(25) (a) Richter, H.; Wang, Z. P.; Ley, L. Solid State Commun. 1981, 39, 625-629. (b) Kanata, T.; Murai, H.; Kubota, K. J. Appl. Phys. 1987, $61,969-971$.

(26) Lipp, M. J.; Baonza, V. G.; Evans, W. J.; Lorenzana, H. E. Phys. Rev. B 1997, 56, 5978-5984.

(27) Zhao, X. S.; Ge, Y. R.; Schroeder, J.; Persans, P. D. Appl. Phys. Lett. 1994, 65, 2033-2035. 


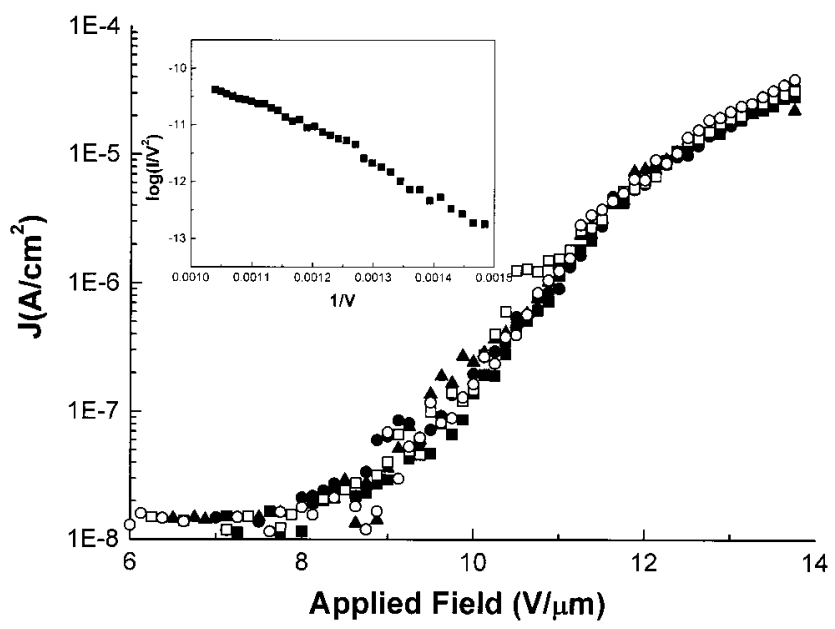

Figure 13. Typical field emission characteristic of the GaN nanowires. The result shows five measurements on the same sample. The $\mathrm{F}-\mathrm{N}$ plot at the inset indicates field emission character of the GaN nanowires.

The relaxation of the $q=0$ selection rule due to the effects of finite crystallite size not only broadens the Raman-allowed modes, but also causes new modes to appear that correspond to $q \neq 0$ phonons. Two extra peaks seen at $\sim 252$ and 421 $\mathrm{cm}^{-1}$ in GaN nanowires could be possibly attributed to the zoneboundary phonons activated by the finite-size effects. In the phonon-dispersion relations for wurtzite $\mathrm{GaN}$, lattice dynamic calculations ${ }^{23 c, 29}$ found that the acoustic, as well as the combination of both acoustic and lower-lying, optical branches at the M symmetry point in the Brillouin zone produce peaks in the phonon density of states near $\sim 250$ and $420 \mathrm{~cm}^{-1}$, respectively. Accordingly, with decreasing size of $\mathrm{GaN}$ crystallites to nanoscale dimensions and increasing the finite-size effects, additional modes are expected to appear at frequencies corresponding to the density of states produced by these zoneboundary phonons. It is worth mentioning that the 252 and 421 $\mathrm{cm}^{-1}$ features were consistently observed at different spots of two GaN nanowire samples grown by using different catalysts. Thus, the possibility of any impurity contaminant effect seems extremely unlikely in the present study. Finally, all spectra reveal a broad band at $\sim 670 \mathrm{~cm}^{-1}$, and its intensity dramatically increases using $\mathrm{He}-\mathrm{Ne}$ laser excitation. This resonant behavior, as well as its relationship to the weak and broad structure near $\sim 2.2 \mathrm{eV}$ in the photoluminescence spectra, suggests that the appearance of the $670 \mathrm{~cm}^{-1}$ mode is likely associated with a defect level below the GaN conduction band minimum. ${ }^{30}$

Field Emission Measurements. Typical field-emission characteristics of the GaN nanowires, specifically emission current density $J$ as a function of applied field, are shown in Figure 13. To determine the current density in the measurement, one needs the effective emission area as well as the total emission current. However, it is difficult to determine the emission area for samples of irregular morphology like nanotubes and nanowires. In this paper, because the anode area is smaller than that

(28) Heath, J. R.; Shiang, J. J.; Alivisatos, A. P. J. Chem. Phys. 1994, 101, 1607-1615.

(29) (a) Siegle, H.; Kaczmarczyk, G.; Filippidis, L.; Litvinchuk, A. P.; Hoffmann, A.; Thomsen, C.Phys. Rev. B 1997, 55, 7000-7004. (b) Bungaro, C.; Rapcewicz, K.; Bernholc, J. Phys. Rev. B 2000, 61, 6720-6725. (c) Tutuncu, H. M.; Srivastava, G. P. Phys. Rev. B 2000, 62, 5028-5035.

(30) Chen, H. H.; Chen, Y. F.; Lee, M. C.; Feng, M. S. Phys. Rev. B 1997, 56, 6942-6946. of sample, the emission current densities were obtained by dividing the measured current by the anode area, assuming homogeneous electron emission from the sample. As shown in Figure 13, the turn-on field of the GaN-nanowires, which is defined as the field required to draw an emission current density of $0.01 \mathrm{~mA} / \mathrm{cm}^{2}$, is $12 \mathrm{~V} / \mu \mathrm{m}$. Moreover, the reproducible result from the five repetitive measurements in Figure 13 indicates that $\mathrm{GaN}$-nanowires exhibit promising field emission properties as well as good stability. The Fowler-Nordheim $(\mathrm{F}-\mathrm{N})$ description of the field emission for metals is expressed as ${ }^{31}$

$$
J=A \frac{F^{2}}{\phi} \exp \left(\frac{-B \phi^{3 / 2}}{F}\right)
$$

where $A=0.014$ and $B=6.8 \times 10^{-9}$ are constants; and $J$ $\left(A / \mathrm{m}^{2}\right)$, the current density; $\phi(\mathrm{eV})$, the effective barrier height; $F=\beta V / d$ with $\mathrm{V}$ (volts), the voltage; $d(\mathrm{~m})$, the cathode-to anode spacing; and $\beta$, the field enhancement factor that depends on the emitter geometry. For materials with field emission characteristics that follow $\mathrm{F}-\mathrm{N}$ description, a plot of $\log \left(\mathrm{I} / \mathrm{V}^{2}\right)$ versus $1 / \mathrm{V}(\mathrm{F}-\mathrm{N}$ plot) would give a straight line. The inset of Figure 13 illustrates the $\mathrm{F}-\mathrm{N}$ plot of the $\mathrm{GaN}$ nanowires. The straight line of the $\mathrm{F}-\mathrm{N}$ plot shows that the emission of the $\mathrm{GaN}$ nanowires follows the field emission behavior. The $20 \mu \mathrm{A} /$ $\mathrm{cm}^{2}$ current density at a field of $14 \mathrm{~V} / \mu \mathrm{m}$ is significant in comparison to other report ${ }^{13}$ for an undoped wide-band-gap material. The field emission property might be further enhanced with proper doping of such nanowires.

In conclusion, high-purity and -quality GaN nanowires were prepared by systematic optimizations of the catalysts, reaction temperatures, total reacting time, and heating rate in the catalytic reaction. The structure and stoichiometry analyses of $\mathrm{GaN}$ nanowires were performed using X-ray diffraction and electron microscopy to illustrate the details of VLS growth of the nanowires. Temperature dependence of photoluminescence spectra of the nanowires indicated that the emission intensity is very resistant to the change of temperature. A very strong luminescence signal arising from band-edge emission of wurtzite $\mathrm{GaN}$ nanowires can be obtained at room temperature. This behavior is particularly useful for the application in optoelectronic devices. Raman-scattering studies of the GaN nanowires reveal five first-order phonon modes near $\sim 144,537,562,569$, and $728 \mathrm{~cm}^{-1}$. The frequencies of these peaks are close to those of the bulk, but significantly broadened, which is indicative of the phonon confinement effects brought out by the nanoscale dimensions of the system. Furthermore, the appearance of two additional modes at $\sim 252$ and $421 \mathrm{~cm}^{-1}$ are attributed to zoneboundary phonons activated by the finite-size effects. Finally, field emission study of the GaN nanowire showed significant emission current at low electric field, indicating potential for vacuum electronics upon additional doping of these nanowires.

Acknowledgment. All authors would like to gratefully acknowledge financial support from the National Science Council and Chinese Petroleum Cooperation in Taiwan. We thank Prof. K. J. Ma, Prof. C. N. Chang, Y. S. Lin, and S. C. Kao for help on electron microscopy measurements and structural analyses.

\section{JA0040518} 548 .

(31) Gadzuk, J. W.; Plummer, E. W. Rev. Mod. Phys. 1973, 45, 487- 